\title{
Isotope gastric emptying tests in clinical practice: expectation, outcome, and utility
}

\author{
M A Galil, M Critchley, C R Mackie
}

\begin{abstract}
Tests of gastric emptying with modern scintigraphic methods are recommended in the clinical management of gastric disorders. An audit of $\mathbf{4 7 2}$ gastric emptying tests carried out over a 10 year period was performed to discover the reasons for requests from consultant clinicians, their anticipation of the results of tests, and the influence of the results upon the subsequent management of their patients. Excluding control $(n=47)$ and research $(n=50)$ studies, there were 375 clinical referrals that could be grouped under the headings: nonulcer dyspepsia $(n=72)$, suspected diabetic gastroparesis $(n=18)$, peptic ulcer $(n=15)$, suspected delayed gastric emptying after surgery $(n=154)$, dumping and diarrhoea $(=107)$, and other indications $(n=9)$. Although the results were abnormal for $55(48 \%)$ of the 'medical' patients, they did not seem to influence clinical management. Delayed gastric emptying after surgery was confirmed in only $20 \%$ of patients referred with this clinical diagnosis. Conversely, most (79\%) of the patients referred with dumping and diarrhoea exhibited abnormally rapid emptying. Isotope gastric emptying studies may be useful in clinical practice. The results are often at variance with the clinical diagnosis. Clinicians must take into account the nature of the test meal used when results are correlated with clinical features.

(Gut 1993; 34: 916-919)
\end{abstract}

University Department of Surgery

M A Gali

C R Mackie

and Department of Nuclear Medicine, Royal Liverpool University Hospital, Liverpool M Critchley

Correspondence to: Mr C R Mackie, Consultant Surgeon, Walton Hospital Rice Lane, Liverpool L9 1AE.

Accepted for publication

10 November 1992
Gastric emptying tests with radiolabelled meals and gamma camera imaging have proved popular for many years in clinical research and physiological studies. ${ }^{1}$ We suspect that departments that have developed gastric emptying test protocols have, as we have, offered a service to clinicians within the region for the assessment of patients with symptoms possibly caused by abnormalities of gastric emptying. We have seen no reports, however, that describe in detail the use of such a service by local clinicians. Many

TABLE I Patients referred after upper gastrointestinal tract surgery

\begin{tabular}{|c|c|c|c|c|}
\hline \multirow[b]{3}{*}{ Previous operations } & \multicolumn{4}{|c|}{ No of patients } \\
\hline & \multirow{2}{*}{$\begin{array}{l}\text { Delayed } \\
\text { emptying } \\
\text { suspected }\end{array}$} & \multicolumn{3}{|c|}{ Rapid emptying suspected } \\
\hline & & Dumping & Diarrhoea & $\begin{array}{l}\text { Dumping and } \\
\text { diarrhoea }\end{array}$ \\
\hline Truncal vagotomy with pyloroplasty & 57 & 22 & 17 & 4 \\
\hline Truncal vagotomy with gastrojejunostomy & 16 & 8 & 13 & 5 \\
\hline Truncal vagotomy with antrectomy & 4 & 1 & 1 & 3 \\
\hline Highly selective vagotomy & 10 & 4 & 1 & 1 \\
\hline Partial gastrectomy & 17 & 10 & 3 & 2 \\
\hline Other operations & 30 & 1 & 4 & 1 \\
\hline Multiple operations & 20 & 2 & i & 3 \\
\hline Total no of patients & 154 & 48 & 40 & 19 \\
\hline
\end{tabular}

articles have appeared ${ }^{2-4}$ that describe potentially useful clinical applications of gastric emptying tests but to discover the clinical value of these tests a comprehensive audit of the service is required.

We believe it would be useful to analyse the sources of referral, the nature of the clinical problems, the suspected abnormalities - rapid or delayed emptying - and whether the result of tests had any significant influence on decisions about treatment.

In this report we present an analysis of 472 gastric emptying studies carried out in the Department of Nuclear Medicine, Royal Liverpool University Hospital between 1981 and 1991 at the request of consultant clinicians in hospitals throughout the Mersey Region. Those studies, the minority, performed as part of clinical trials or formal clinical assessments are indicated as such as mentioned only for completeness. The analysis has been achieved by detailed review of hospital case sheets. Referring clinicians gave permission for this to be carried out and the study was approved by the local Hospital Ethical Committee.

\section{Patients and methods}

Copies of reports of 472 gastric emptying studies performed in the Department of Nuclear Medicine, Royal Liverpool University Hospital between 1981 and 1991 were reviewed. There were 47 control studies, 50 research studies (no clinical diagnostic problem), and 375 studies carried out as part of the clinical service offered by the Department, at the request of consultant clinicians. The hospital case notes of all referred patients were reviewed in detail, and the following information noted: (1) the referring Hospital; (2) the referring clinical discipline; (3) the patient's symptoms and 'working' diagnosis; (4) whether the clinician's notes, before the gastric emptying study, indicated a prediction of abnormally rapid or abnormally slow gastric emptying; (5) the influence of the result of the gastric emptying study upon the subsequent clinical management.

Unless otherwise requested gastric emptying was assessed using a standard physiological test meal incorporating technetium-99m $\left({ }^{99 m} \mathrm{Tc}\right)$ labelled bran and semisolid in character. ${ }^{56}$ When specifically requested, emptying of liquid was assessed using $15 \%$ dextrose labelled with ${ }^{99} \mathrm{~m}$ Tcdiethylene triamine penta-acetic acid.

\section{Results}

The normal range of responses to testing was taken from 47 studies carried out on normal healthy volunteers. Details of these studies have 
been reported previously. ${ }^{5-7}$ The semisolid test meal ( 37 subjects) was found to have a mean half emptying time of 45 minutes with no appreciable lag phase. ${ }^{56}$ For the liquid test meal (10 subjects) the mean emptying at 15 minutes was $10 \% .^{7}$ For both tests, data from control studies were used to derive $95 \%$ confidence intervals for all points on the emptying curves for normal subjects, which were then used to assign normality or abnormality to the results of subsequent studies. Three types of abnormal results were recognised and all abnormal results were judged to be one of these types: rapid emptying, slow emptying, or biphasic emptying (early rapid emptying followed by normal or slow emptying).

Thirty six patients with peptic ulceration were studied before and after operation, as part of a research protocol. Similarly, there were nine patients who were studied after pylorus preserving pancreaticoduodenectomy, and five patients with a diagnosis of achalasia of the cardia. For these patients there was no doubt about the diagnosis or unexplained symptoms. These patients have been excluded from further analysis. Other patients whose studies were part of a research protocol have been included in the subsequent analysis as these requests for gastric emptying studies were intended to establish diagnoses, albeit to define groups of patients for further evaluation. There were therefore, 375 patients referred for diagnostic purposes.

Patients referred for diagnostic studies were grouped according to their symptoms, whether symptoms developed after operation, and according to the stated anticipation of the result, slow or fast emptying, recorded by the referring clinician.

'NON-ULCER DYSPEPSIA': NO PREVIOUS SURGERY Seventy two patients (ages 16-72) with unexplained dyspeptic symptoms of duration six months to seven years were referred by medical (52 patients) and surgical ( 20 patients) consultants. All received the standard semisolid test meal. The result was normal for $39(54 \%)$ patients, rapid for 17 (24\%), biphasic (early rapid then normal) for four (5\%), and slow for 12 (17\%) patients. The results did not seem to influence subsequent clinical management of these patients in any way that would have differed appreciably from management without this information.

SUSPECTED DIABETIC GASTROPARESIS

Eighteen diabetic patients (ages 22-70) with upper gastrointestinal tract symptoms, mostly anorexia and vomiting, were referred by consult-

TABLE II Patients with suspected delayed emptying after upper gastrointestinal tract surgery: abnormalities identified by barium studies and endoscopy

\begin{tabular}{lc}
\hline Abnormality & No of patients \\
\hline Gastritis & 10 \\
Enterogastric bile reflux & 10 \\
Delayed gastric emptying & 8 \\
Gastric outlet obstruction & 27 \\
Gastro-oesophageal reflux & 6 \\
Gastric Crohn's disease & 1 \\
No abnormality shown & 92 \\
\hline
\end{tabular}

ant physicians ( 16 patients) and studied using the semisolid test meal. Gastric emptying was normal or biphasic in eight patients, rapid in seven, and delayed in three patients.

\section{PATIENTS WITH PEPTIC ULCER}

Fifteen peptic ulcer patients who were not part of any formal trial of treatment, were referred, chiefly by surgeons (12 patients) presumably to help choose the most appropriate operation. Studies using the semisolid test meal showed normal gastric emptying in six patients, normal emptying after a rapid early phase in five patients, uniformly rapid emptying in three, and slow emptying in one patient. Only the result for this last patient is noted to have influenced the choice of operation.

\section{SUSPECTED DELAYED GASTRIC EMPTYING AFTER} SURGERY

There were 154 patients (ages 20-83) complaining of vomiting and epigastric pain or discomfort after various upper gastrointestinal tract operations (Table I). Clinically delayed gastric emptying was suspected. These patients were referred more commonly by surgeons (127 patients) than physicians (27 patients) and had had symptoms for between two months and six years. Gastroscopy and barium studies had shown various 'abnormalities' of uncertain significance (62 patients - Table II), or no abnormality (92 patients). Gastric emptying studies were carried out using the $15 \%$ dextrose test meal for 16 patients and the semisolid test meal for 138 patients. Gastric emptying was confirmed to be abnormally slow in $31(20 \%)$ patients. Emptying was normal in $27(17 \%)$ patients, and either uniformly rapid (31 patients $-20 \%$ ) or biphasic with an early rapid phase (65 patients $-42 \%$ ) in the remainder. Ultimately, $10(6 \%)$ patients from within the whole group had further surgery the nature of which seemed to have been influenced by the results of the test. Six patients had planned operations cancelled because of the test result. For 98 (64\%) patients there was no recorded indication that the result of the test influenced subsequent management.

\section{DUMPING AND DIARRHOEA}

One hundred and seven patients, 102 (95\%) of whom had had a vagotomy or partial gastrectomy (Table I), were referred because of suspected rapid gastric emptying as a cause or feature of dumping syndrome and diarrhoea, or both (Table I). Sixteen (15\%) of these patients were referred by physicians, the rest by surgeons. Sixty eight $(63 \%)$ patients were men and ages ranged from 22 to 73 years. Duration of symptoms varied from two months to 15 years. Nineteen (18\%) patients were studied using $15 \%$ dextrose, while $88(82 \%)$ patients were assessed using the semisolid test meal. Gastric emptying was shown to be very rapid or biphasic with a rapid early component in 85 (79\%) patients, normal in 14 $13 \%$ ), and abnormally slow in eight (7\%) patients. Within the whole group, according to the case records, revisional surgery plan- 
TABLE III Patterns of referral for gastric emptying studies

\begin{tabular}{|c|c|c|c|c|c|c|}
\hline \multirow[b]{4}{*}{ Patient group } & \multicolumn{6}{|c|}{ No of patients } \\
\hline & \multirow[b]{3}{*}{ Total } & \multicolumn{3}{|c|}{ Referring hospital } & \multirow{2}{*}{\multicolumn{2}{|c|}{ Referring discipline }} \\
\hline & & \multirow[b]{2}{*}{ University } & \multicolumn{2}{|c|}{ District general } & & \\
\hline & & & Central & Peripheral & Surgical & Medical \\
\hline Non-ulcer dyspepsia & 72 & 19 & 37 & 16 & 20 & 52 \\
\hline Suspected diabetic gastroparesis & 18 & 12 & 3 & 3 & 2 & 16 \\
\hline Peptic ulcer & 15 & 8 & 6 & 1 & 12 & 3 \\
\hline Suspected delayed emptying after & & & & & & \\
\hline surgery & 154 & 51 & 94 & 9 & 127 & 27 \\
\hline Dumping and diarrhoea, or both & 107 & 40 & 56 & 11 & 91 & 16 \\
\hline Other indications & 9 & 3 & 5 & 1 & 5 & 4 \\
\hline
\end{tabular}

ned before the study was cancelled as a result of the study in two cases. Twelve patients had revisional surgery and it seems likely that the results of tests helped with the treatment decisions for these patients.

\section{OTHER INDICATIONS}

Abnormal results were obtained for three of nine other patients referred for gastric emptying tests (systemic sclerosis - 3 patients, gastrooesophageal reflux -2 patients, distal pancreatectomy -2 patients, adult hypertrophic pyloric stenosis - 1 patient, chronic renal failure 1 patient). Table III shows the pattern of referral from different types of hospital and the two main disciplines. Overall, $72 \%$ of referrals were from surgeons and $64 \%$ of referred patients were men.

\section{Discussion}

In this study the number and type of referrals, and the test methods and results have been obtained from contemporaneous files of duplicate request and result forms kept in the Department of Nuclear Medicine in which complete records are held. Information obtained retrospectively from case notes is unreliable but generally the correspondence to be found in case notes showed the clinician's reason for referring a patient and gave reliable clues to the prediction of the result. We are well aware, however, that the results of this study do not form a basis for firm conclusions about the pathophysiology of specific clinical disorders.

Surgical referrals outnumbered medical referrals by about three to one, a reflection of the predominance of surgically induced abnormalities of gastric emptying among all patients with clinically recognised disorders of gastric emptying. Not surprisingly, the greatest number of referrals per total inpatient bed numbers were from the Royal Liverpool University Hospital, where there is the facility to carry out gastric emptying tests on site. A similar number of referrals came, however, from nearby large district general hospitals. Fewer patients were referred from peripheral hospitals in the region, even though one would expect the many peripheral hospitals jointly to have seen as many patients with similar problems as the fewer large central district general hospitals.

The results of tests carried out on patients with non-ulcer dyspepsia illustrate the limited role for tests of gastric emptying in the management of patients with symptoms not attributable to pre- vious surgery. Although many patients in this group had abnormal results, both fast and slow emptying, the characterisation of an abnormality of gastric emptying in a patient with non-ulcer dyspepsia does not necessarily provide a key to effective treatment. It cannot be assumed that symptoms are caused by disturbed gastric emptying. In practice, moreover, treatments for such patients tend to be chosen empirically and worked through on a trial and error basis, whatever might be thought to be the underlying pathophysiology.

The results for patients suspected of having diabetic gastroparesis - more patients with rapid than with slow emptying - might seem surprising but illustrates an important consideration that is too easily overlooked. Rates of gastric emptying vary enormously in the same individual depending on the nature of the test meal studied. Overall, the most significant abnormality seems to be a divergence of the emptying rates of liquids and solids with liquids emptying faster than normal and solids being retained in the stomach for longer than normal. The 'semisolid' test meal used in our studies behaves as a slow emptying liquid. Thus there is no appreciable lag phase before emptying and the curve is non-linear. In the diabetic subjects studied, therefore, the possibility of gastric retention of solids has not been answered.

There may be confusion in the clinical diagnosis of delayed gastric emptying after upper gastrointestinal tract surgery, and there are traps for the unwary. Often, the vomiting of food may be thought to point to the presence of a simple, partial mechanical obstruction to gastric emptying. Usually, however, the situation is more complicated than this; in such patients gastric emptying of liquid, as this study shows, may be abnormally rapid, while concurrent retention of solids suggests the maintenance of antral sieving with failure or inhibition of effective trituration. Gastric emptying studies with a truly solid meal could be helpful in the assessment of these patients. Such a test has not been routinely available in our Department and this could be seen as a shortcoming in the service that has been offered, as well as a limitation to the scope of this study. Nevertheless, it seems worth pointing out that gross gastric retention of solids can usually be confirmed quite easily by gastroscopy after a six hour fast, while the key gastric emptying study, with liquid or liquid like test meal, is applicable in postoperative patients with vomiting and epigastric pain after meals to confirm that there is no mechanical obstruction, and to warn that a further simple gastric drainage procedure may do more harm than good.

An attempt to determine before operation whether peptic ulcer patients are at risk of developing postvagotomy symptoms seems laudable but in practice such clues as there may be are probably too subtle to identify with any confidence from a non-invasive gastric emptying test. Perhaps a more fruitful method should involve direct intraduodenal instillation of nutrient liquid in response to which some subjects may develop signs and symptoms of vasomotor dumping.

Most patients clinically suspected of dumping 
or diarrhoea after having a vagotomy or gastrectomy did indeed exhibit abnormally rapid gastric emptying. It seems prudent to confirm that such patients do have rapid gastric emptying, particularly if remedial surgery is being considered. Unfortunately, most patients exhibit abnormally rapid gastric emptying of liquids after truncal vagotomy or partial gastrectomy irrespective of whether or not they have symptoms, and there is considerable overlap of results between symptomatic and asymptomatic groups. ${ }^{9}$ Gastric emptying tests cannot therefore be used to make the diagnosis, although they can, nevertheless yield results consistent with the diagnosis. A test before remedial surgery can, in addition, be useful when reassessment, after further operation, is required.

We believe that the results of this survey help to highlight the clinical circumstances in which tests of gastric emptying may be useful. Patients with 'non-ulcer dyspepsia' who seem refractory to all treatments might be assessed in this way to ensure they do not have a gross disorder of gastric emptying, but the likelihood that the result will advance clinical management seems small. Patients with vomiting of food after upper gastrointestinal tract surgery should be studied if there is doubt, after an endoscopy and barium studies or both whether they have some form of mechanical obstruction, but usually they do not. For patients with postoperative dumping or diarrhoea it is prudent to assess gastric emptying before starting remedial surgery. If the patient's symptoms do not warrant such treatment, there seems little point in requesting studies; the result is likely to show rapid emptying of liquids even if the symptoms are only mild.

Dr M A Galil is in receipt of a British Council Scholarship.

1 Griffith GH, Owen GM, Kirkman S, Shields R. Measurement of rate of gastric emptying using Chromium-51. Lancet 1966; i: $1244-5$.

2 Dubois A. Pathophysiology of gastric emptying: Methods of measurement and clinical significance. $\mathcal{F}$ Clin Gastroenterol $1979 ; 1: 259-66$.

3 Malagelada J-R. Physiologic basis and clinical significance of gastric emptying disorders. Dig Dis Sci 1979; 24: 657-61

4 Pellegrini CA, Broderick WC, Van Dyke D, Way LW. Diagnosis and treatment of gastric emptying disorders. Am $\dot{\mathfrak{F}}$ Surg 1983; 145: 143-51.

5 Sagar S, Grime JS, Little W, et al. Technetium-99m labelled bran: a new agent for measuring gastric emptying. Clin Radiol 1983; 34: 275-8

6 Baxter JN, Grime JS, Critchley M, Shields R. Relationship between gastric emptying of solids and gall bladder emptying in normal subjects. Gut 1985; 26: 342-51.

7 Parr NJ, Grime S, Brownless S, Critchley M, Baxter JN, Mackie CR. Relationship between gastric emptying of liquid and postvagotomy diarrhoea. Br $\mathcal{F}$ Surg 1988; 75: 279-82.

8 Snook JA, Wells AD, Prytherch DR, Evans DH, Bloom SR, Colin-Jones DG. Studies on the pathogenesis of the early dumping syndrome induced by intraduodenal instillation of dumping syndrome induced by intraduode

hypertonic glucose. Gut 1989; 30: 1716-20.
9 Ralphs DNL, Thomson JPS, Haynes S, Lawson-Smith C, Hobsley M, Le Quesne LP. The relationship between the rate of gastric emptying and the dumping syndrome. $\mathrm{Br} \mathcal{F}$ Surg 1978; 76: 637-41. 